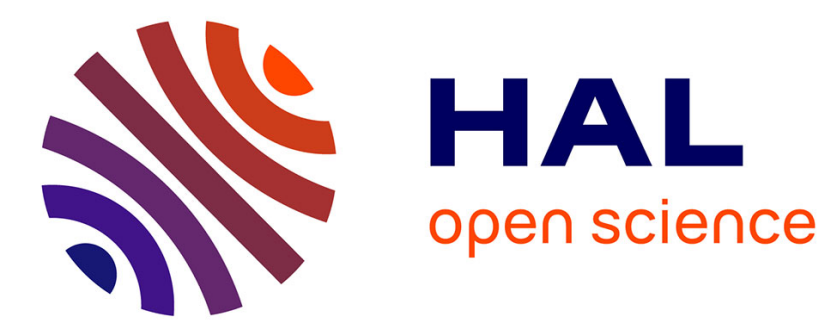

\title{
Probabilistic model of the human cortical bone with mechanical alterations in ultrasonic range
}

Christophe Desceliers, Christian Soize, Salah Naili, Guillaume Haïat

\section{To cite this version:}

Christophe Desceliers, Christian Soize, Salah Naili, Guillaume Haïat. Probabilistic model of the human cortical bone with mechanical alterations in ultrasonic range. Mechanical Systems and Signal Processing, 2012, 32 (-), pp.170-177. 10.1016/j.ymssp.2012.03.008 . hal-00718959

\section{HAL Id: hal-00718959 https://hal.science/hal-00718959}

Submitted on 19 Oct 2012

HAL is a multi-disciplinary open access archive for the deposit and dissemination of scientific research documents, whether they are published or not. The documents may come from teaching and research institutions in France or abroad, or from public or private research centers.
L'archive ouverte pluridisciplinaire $\mathbf{H A L}$, est destinée au dépôt et à la diffusion de documents scientifiques de niveau recherche, publiés ou non, émanant des établissements d'enseignement et de recherche français ou étrangers, des laboratoires publics ou privés. 


\title{
Probabilistic model of the human cortical bone with mechanical alterations in ultrasonic range
}

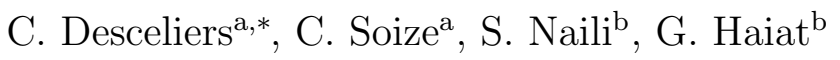 \\ ${ }^{a}$ Université Paris-Est, Laboratoire Modélisation et Simulation Multi Echelle, \\ MSME UMR 8208 CNRS, \\ 5 bd Descartes, 77454 Marne-la-Vallee, France \\ ${ }^{b}$ Université Paris-Est, Laboratoire Modélisation et Simulation Multi Echelle, \\ MSME UMR 8208 CNRS, \\ 61 avenue du Général de Gaulle, 94010 Créteil Cedex, France
}

\begin{abstract}
The biomechanical materials are among the most complex mechanical systems. Most often, their microstructure are heterogeneous and random. This is the case for the human cortical bones which are considered in this paper. For such systems, a gradient of porosity can be observed in the thickness direction but, in this case, none of the usual theories of porous materials can be applied. For this reason, a simplified model with gradient for the elasticity tensor is presented. The predictability of this model is improved by taking into account uncertainties. We propose a prior stochastic model of the tensor-valued elasticity field corresponding to an extension of a previous work in which the random elasticity field was constant in space. This extension consists in introducing two ingredients: the introduction of a spatial gradient for the mean elasticity tensor and spatial statistical fluctuations in the thickness direction. The stochastic model which is constructed shows that
\end{abstract}

\footnotetext{
${ }^{*}$ Corresponding author

Email address: christophe.desceliers@univ-paris-est.fr(C. Desceliers)
} 
the observed responses are effectively sensitive with respect to the values of the gradient and to the level of statistical fluctuations. In this sense, this stochastic model will be well adapted to perform its identification solving a stochastic inverse problem that is not the purpose of present paper.

Keywords:

multilayer, mesocale, gradient of properties, elasticity tenor, uncertainties

\section{Introduction}

The aim of this paper is to introduce a stochastic elastoacoustic model useful for the ultrasonic characterization of a multilayer materials for which an elastic solid layer is uncertain. This stochastic model is developed in the context of the ultrasonic propagation in human cortical bones but could be applied to another mechanical systems. The biomechanical materials are among the most complex mechanical systems. Modeling such media is a challenge and the main difficulty is given rise to the complexity level of their microstructures. This is the case for the human cortical bones which are considered in this paper. The complexity level of such a biomechanical system is such that a multiscale approach should be developed to represent all the mechanical behavior and would allow (among others) the ultrasonic characterization of a human compact bone to be simulated. Nevertheless, such a multiscale model would be very difficult to construct taking into account the complexity of the heterogeneities of the microstructure. In this paper, we are only interested in the ultrasonic characterization of the human cortical bone. In this context, a first simplified model has been introduced in [16, 9] and which is representative of in vivo measurements of bone cortical properties 
with the so-called axial transmission technique [13]. This simplified elastoacoustic model is a three-layers system: an elastic solid layer sandwiched between two acoustic fluid layers. The elastic solid layer (cortical bone) is an anisotropic elastic material while the fluid acoustic layers (soft tissues) are usual compressible and homogenous inviscid fluids satisfying the linear acoustic equations. The axial transmission technique provides the velocity of ultrasonic waves axially transmitted along cortical bone through a linear arrangement of transmitters and receivers placed on the same side of the skeletal site. This technique has been extensively used to probe bone quality at the radius $[4,8]$. With the bidirectional axial transmission technique, the measurements of the velocity of the first arriving signal (FAS) is sensitive to the elasticity properties of the cortical bone in the neighborhood of the coupling interface between the elastic solid layer (cortical bone) and the acoustic fluid layer (soft tissues). Nevertheless, previous works [5, 6, 10, 17] shows that the FAS is not sensitive to the bone mass density and to the bone thickness when this one is greater than the wavelength in the cortical bone. This is a reason why, in this paper, an extension of the stochastic model and an additional observation are introduced in order to also obtain a sensitivity with respect to the bone thickness. It should be noted that the in vivo measurements [9] exhibit random fluctuations of the ultrasonic characterization which are not related to measurement errors induced by the probe. In Desceliers et al. [9], it was seen that a simplified mechanical model with an additional stochastic modeling of bone elasticity properties are able to represent the in vivo measurements in the statistical sense. It should be noted that a first application of the parametric probabilistic approach to the 
axial transmission technique can be found in Macocco et al. [14].

Besides its multiscale nature, cortical bone is heterogeneous at the organ scale. Its mechanical properties depend on the cross-sectional and axial location $[19,20]$. This heterogeneity depends on properties such as porosity and degree of mineralization of bone tissue [18]. In particular, porosity in the radial direction (which is associated with the cross-section) is heterogeneous at all ages and for both genders [7]. In this paper, for such a biomechanical system, we consider that the microstructure of the cortical bone is altered in the neighborhood of its interface with the marrow. As a result, strong fluctuations of the pore sizes can exist in the thickness direction but, in this case, none of the usual theories of porous materials $[1,2,3]$ can be applied because the variations of the pore sizes are too much important with respect to the number of pores and consequently, homogenization cannot be applied. Consequently, in the thickness direction, the spatial variation of the mean elasticity properties for the mean model at the mesoscale modeling of the microstructure, is taken into account in introducing a gradient of the elasticity properties. The determination of the gradient of the mean elasticity properties is of a first interest in order to diagnose bone diseases through the quantification of bone stiffness. Thus, inside the elastic solid layer (cortical bone), the constitutive equation goes to the one of the acoustic fluid (marrow) when one moves towards the marrow in the thickness direction. The uncertainties related to such a model are taken into account by modeling the elasticity tensor by a random field for which the mean value corresponds to the previous mean model exhibiting a gradient. 
In a first step, the simplified mean model introduced in Desceliers et al. [9] is briefly recalled. The three next sections are devoted to the construction of the gradient model for the mean elasticity properties of the microstructure at the mesoscale. In the following section, the stochastic model around the mean model is constructed. Finally, we introduce a new observation allowing the determination of the parameters of the probabilistic model to be performed and we present a numerical illustration for the cortical bone.

\section{Simplified model}

The properties of the human cortical bone are studied by using in vivo measurements obtained with the axial transmission technique. An acoustic pulse is applied to the skin layer in the ultrasonic range and the velocity of the first arriving signal is measured. A simplified model of the human cortical bone made up of the skin, the coupling gel with a probe which generated an acoustic pulse and the marrow has been developed in Naili et al. [16], Desceliers et al. [9]. This simplified model is composed of an elastic solid semi-infinite layer between two acoustic fluid semi-infinite layers as shown in Fig. 1.

Let $\mathbf{R}\left(O, \mathbf{e}_{1}, \mathbf{e}_{2}, \mathbf{e}_{3}\right)$ be the reference Cartesian frame where $O$ is the origin of the space and $\left(\mathbf{e}_{1}, \mathbf{e}_{2}, \mathbf{e}_{3}\right)$ is an orthonormal basis. The generic point in $\mathbb{R}^{3}$ is $\mathbf{x}=\left(x_{1}, x_{2}, x_{3}\right)$. The thicknesses of the layers are denoted by $h_{1}, h$ and $h_{2}$ (see Fig. 1). The first acoustic fluid layer occupies the unbounded domain $\Omega_{1}$, the second one occupies the unbounded domain $\Omega_{2}$ and the elastic solid layer occupies the unbounded domain $\Omega$. Let $\partial \Omega_{1}=\Gamma_{1} \cup \Sigma_{1}, \partial \Omega=\Sigma_{1} \cup \Sigma_{2}$ 


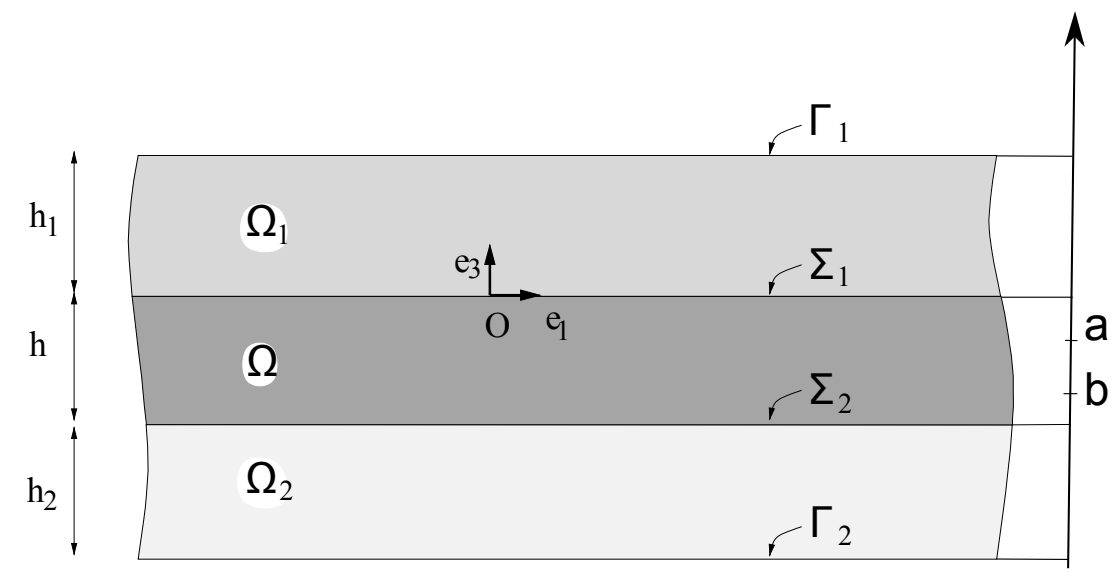

Figure 1: Geometry of the multilayer system

and $\partial \Omega_{2}=\Sigma_{2} \cup \Gamma_{2}$ (see Fig. 1) be the boundaries of $\Omega_{1}, \Omega$ and $\Omega_{2}$ in which $\Gamma_{1}, \Sigma_{1}, \Sigma_{2}$ and $\Gamma_{2}$ are the planes defined by

$$
\begin{gathered}
\Gamma_{1}=\left\{x_{1} \in \mathbb{R}, \quad x_{2} \in \mathbb{R}, x_{3}=h_{1}\right\}, \\
\Sigma_{1}=\left\{x_{1} \in \mathbb{R}, \quad x_{2} \in \mathbb{R}, x_{3}=0\right\}, \\
\Sigma_{2}=\left\{x_{1} \in \mathbb{R}, \quad x_{2} \in \mathbb{R}, x_{3}=-h\right\}, \\
\Gamma_{2}=\left\{x_{1} \in \mathbb{R}, \quad x_{2} \in \mathbb{R}, x_{3}=-\left(h+h_{2}\right)\right\} .
\end{gathered}
$$

Let $\mathbf{n}$ be the outward unit normal to domain $\Omega$. Let $\mathbf{u}$ be the displacement field of the elastic solid layer occupying the domain $\Omega, p_{1}$ be the acoustic pressure field of the acoustic fluid layer occupying domain $\Omega_{1}$ and $p_{2}$ be the acoustic pressure field of the one occupying domain $\Omega_{2}$. It should be noted that domains $\Omega_{1}, \Omega$ and $\Omega_{2}$ are unbounded along the transversal directions $\mathbf{e}_{1}$ and $\mathbf{e}_{2}$ whereas they are bounded along the vertical direction $\mathbf{e}_{3}$.

At time $t=0$, the system is assumed to be at rest. 


\section{Mean boundary value problem for the first acoustic fluid layer}

A line source modeling the acoustical impulse is applied in domain $\Omega_{1}$ and is defined by a source density $S$ such that

$$
\frac{\partial S}{\partial t}(\mathbf{x}, t)=\rho_{0,1} F(t) \delta_{0}\left(x_{1}-x_{1}^{S}\right) \delta_{0}\left(x_{3}-x_{3}^{S}\right)
$$

in which $F(t)=F_{1} \sin \left(2 \pi f_{c} t\right) e^{-4\left(t f_{c}-1\right)^{2}}$ where $f_{c}=1 \mathrm{MHz}$ is the central frequency, $F_{1}=100 \mathrm{~N}, \rho_{0,1}$ is the mass density of the acoustic fluid in domain $\Omega_{1}, \delta_{0}$ is the Dirac function at the origin, $x_{1}^{S}$ and $x_{3}^{S}$ are the coordinates of the line source. It can be shown that, for all $\mathbf{x}$ belonging to $\Omega_{1}$ and for all time $t>0$, acoustic pressure field $p_{1}$ verifies

$$
\begin{gathered}
\frac{1}{K_{1}} \frac{\partial^{2} p_{1}}{\partial t^{2}}-\frac{1}{\rho_{1,0}} \Delta p_{1}=\frac{1}{\rho_{1,0}} \frac{\partial S}{\partial t} \quad, \mathbf{x} \in \Omega_{1} \\
p_{1}=0 \quad, \mathbf{x} \in \Gamma_{1} \\
\operatorname{grad} p_{1} \cdot \mathbf{n}=-\rho_{1,0} \frac{\partial^{2} \mathbf{u}}{\partial t^{2}} \cdot \mathbf{n} \quad, \mathbf{x} \in \Gamma_{0}
\end{gathered}
$$

in which $K_{1}=\rho_{1,0} c_{1,0}^{2}$, where $c_{1,0}$ and $\rho_{1,0}$ are respectively the wave velocity in the fluid and the mass density of the fluid at equilibrium; The symbol $\Delta$ is the Laplacian operator with respect to $\mathbf{x}$.

\section{Mean boundary value problem for the elastic solid layer}

The elastic solid displacement field $\mathbf{u}$ for the mean model verifies

$$
\begin{gathered}
\rho \frac{\partial^{2} \mathbf{u}}{\partial t^{2}}-\operatorname{div} \sigma=\mathbf{0} \quad, \mathbf{x} \in \Omega \\
\sigma \mathbf{n}=-p_{1} \mathbf{n} \quad, \mathbf{x} \in \Sigma_{1} \\
\sigma \mathbf{n}=-p_{2} \mathbf{n} \quad, \mathbf{x} \in \Sigma_{2}
\end{gathered}
$$


in which $\rho\left(x_{3}\right)$ is the mass density which is assumed to be independent of $x_{1}$ and $x_{2}$ at any point $\mathbf{x}$ (we only consider heterogeneities of the material in the thickness direction). The quantity $\sigma(\mathbf{x}, t)$ is the Cauchy stress tensor of the elastic solid layer at point $\mathbf{x}$ and at time $t>0$ and $p_{2}(\mathbf{x}, t)$ denotes the acoustic pressure field in the fluid occupying the domain $\Omega_{2}$; The symbol div is the divergence operator with respect to $\mathbf{x}$. The constitutive equation of the elastic solid layer is written as

$$
\sigma(\mathbf{x}, t)=\sum_{i, j, k, h=1}^{3} c_{i j k h}\left(x_{3}\right) \varepsilon_{k h}(\mathbf{x}, t) \mathbf{e}_{i} \otimes \mathbf{e}_{j}
$$

where $\left\{c_{i j k h}\left(x_{3}\right)\right\}$ is the mean elasticity tensor of the fourth order at the mesoscale of the microstructure, which is assumed to be independent of $x_{1}$ and $x_{2}$ at any point $\mathbf{x}$ (we only consider heterogeneities of the material in the thickness direction). The symbol $\otimes$ designates the tensor product between two vectors. The quantity $\varepsilon_{k h}=\frac{1}{2}\left(\frac{\partial u_{k}}{\partial x_{h}}+\frac{\partial u_{h}}{\partial x_{k}}\right)$ is the linearized strain tensor. It is usual to associate the elasticity $6 \times 6$ matrix $\left[C\left(x_{3}\right)\right]$ to elasticity tensor $\left\{c_{i j k h}\left(x_{3}\right)\right\}$.

If the mean model at mesoscale was a homogeneous transverse isotropic material with a constant mass density $\rho\left(x_{3}\right)=\rho^{S}$, then $\left[C\left(x_{3}\right)\right]$ would be equal to a constant matrix $\left[C^{S}\right]$ whose entries are equal to zero except the following one:

$$
\begin{gathered}
{\left[C^{S}\right]_{11}=\frac{e_{L}^{2}\left(1-\nu_{T}\right)}{\left(e_{L}-e_{L} \nu_{T}-2 e_{T} \nu_{L}^{2}\right)} \quad, \quad\left[C^{S}\right]_{22}=\frac{e_{T}\left(e_{L}-e_{T} \nu_{L}^{2}\right)}{\left(1+\nu_{T}\right)\left(e_{L}-e_{L} \nu_{T}-2 e_{T} \nu_{L}^{2}\right)}} \\
{\left[C^{S}\right]_{12}=\frac{e_{T} e_{L} \nu_{L}}{\left(e_{L}-e_{L} \nu_{T}-2 e_{T} \nu_{L}^{2}\right)} \quad, \quad\left[C^{S}\right]_{23}=\frac{e_{T}\left(e_{L} \nu_{T}+e_{T} \nu_{L}^{2}\right)}{\left(1+\nu_{T}\right)\left(e_{L}-e_{L} \nu_{T}-2 e_{T} \nu_{L}^{2}\right)}} \\
{\left[C^{S}\right]_{44}=g_{T} \quad, \quad\left[C^{S}\right]_{55}=g_{L},}
\end{gathered}
$$


with $\left[C^{S}\right]_{22}=\left[C^{S}\right]_{33},\left[C^{S}\right]_{12}=\left[C^{S}\right]_{13}=\left[C^{S}\right]_{21}=\left[C^{S}\right]_{31},\left[C^{S}\right]_{23}=\left[C^{S}\right]_{32}$ and $\left[C^{S}\right]_{55}=\left[C^{S}\right]_{66}$; the quantities $e_{L}$ and $e_{T}$ are the longitudinal and transversal Young moduli, $g_{L}$ and $g_{T}$ are the longitudinal and transversal shear moduli and $\nu_{L}$ and $\nu_{T}$ are the longitudinal and transversal Poisson coefficients such that $g_{T}=e_{T} / 2\left(1+\nu_{T}\right)$.

\section{Mean boundary value problem for the second acoustic fluid layer}

Let $\mathbf{v}_{2}(\mathbf{x}, t)$ be the velocity field of a particle located at point $\mathbf{x}$ of $\Omega_{2}$ and at time $t>0$. The corresponding displacement field is denoted by $\mathbf{u}_{2}(\mathbf{x}, t)$. We then have $\mathbf{v}_{2}=\partial \mathbf{u}_{2} / \partial t$. For all $\mathbf{x}$ belonging to $\Omega_{2}$ and for all time $t>0$, the mass density of the acoustic fluid layer occupying domain $\Omega_{2}$ is denoted by $\rho_{2}(\mathbf{x}, t)$. In the context of the linear acoustic theory, the linearized mass conservation equation is written as

$$
\frac{\partial \rho_{2}}{\partial t}+\rho_{2,0} \operatorname{div} \mathbf{v}_{2}=0 \quad, \mathbf{x} \in \Omega_{2}
$$

in which $\rho_{2,0}$ is the mass density of the fluid at equilibrium. Furthermore, the linearized Euler equation is written as

$$
\rho_{2,0} \frac{\partial \mathbf{v}_{2}}{\partial t}+\operatorname{grad} p_{2}=\mathbf{0} \quad, \mathbf{x} \in \mathbf{\Omega}_{\mathbf{2}}
$$

in which grad denotes the gradient operator with respect to $\mathbf{x}$. Let $c_{2,0}$ be the wave velocity in the fluid at equilibrium. The constitutive equation for the acoustical fluid occupying domain $\Omega_{2}$ is then written as

$$
p_{2}=c_{2,0}^{2} \rho_{2} \quad, \mathbf{x} \in \Omega_{2}
$$


Then, it can be shown that acoustic pressure field $p_{2}$ satisfies the usual following equation

$$
\begin{gathered}
\frac{1}{K_{2}} \frac{\partial^{2} p_{2}}{\partial t^{2}}-\frac{1}{\rho_{2,0}} \Delta p_{2}=0 \quad, \mathbf{x} \in \Omega_{2} \\
p_{2}=0 \quad, \mathbf{x} \in \Gamma_{2} \\
\operatorname{grad} p_{2} \cdot \mathbf{n}=-\rho_{2,0} \frac{\partial^{2} \mathbf{u}}{\partial t^{2}} \cdot \mathbf{n} \quad, \mathbf{x} \in \Gamma
\end{gathered}
$$

in which $p_{2}(\mathbf{x}, t)$ denotes the acoustic pressure field in the fluid and $K_{2}=$ $\rho_{2,0} c_{2,0}^{2}$. Moreover, consider an elastic solid layer whose constitutive equation is written as $\left\{\sigma_{F}(\mathbf{x}, t)\right\}_{i j}=\sum_{k, h=1}^{3} c_{F, i j k h} \varepsilon_{F, k h}(\mathbf{x}, t)$ where $\left\{\sigma_{F}(\mathbf{x}, t)\right\}_{i j}$ and $\varepsilon_{F, k h}(\mathbf{x}, t)$ are respectively the Cauchy stress and the linearized strain tensors associated with displacement field $\mathbf{u}_{2}$. The elasticity tensor of fourth order $c_{F, i j k h}$ is such that

$$
c_{F, i j k h}=\rho_{0,2} c_{2,0}^{2} \delta_{i j} \delta_{k h}
$$

We then have $\left\{\sigma_{F}(\mathbf{x}, t)\right\}_{i j}=\left(c_{2,0}^{2} \rho_{0,2} \operatorname{div} \mathbf{u}_{2}\right) \delta_{i j}=-\left(p_{F}(\mathbf{x}, t)\right) \delta_{i j}$ in which $p_{F}=-c_{2,0}^{2} \rho_{0,2} \operatorname{div} \mathbf{u}_{2}$ is the acoustic pressure field in the medium. We then have $\partial p_{F} / \partial t=-c_{2,0}^{2} \rho_{0,2} \operatorname{div} \mathbf{v}_{2}$ and using Eq. (9), it can be deduced that $\partial p_{F} / \partial t=c_{2,0}^{2} \partial \rho_{2} / \partial t$ and consequently, $p_{F}=c_{2,0}^{2} \rho_{2}$ (since the acoustic pressure field is equal to zero when the system is at rest). As a consequence, the constitutive equation of such an elastic solid is equivalent to the constitutive equation given by Eq. (11) of an acoustic fluid. In addition, the balance equation for such an elastic solid would be

$$
\rho_{2} \frac{\partial^{2} \mathbf{u}_{2}}{\partial t^{2}}-\operatorname{div} \sigma_{F}=\mathbf{0} \quad, \mathbf{x} \in \Omega
$$

where $\left\{\operatorname{div} \sigma_{F}\right\}_{i}=\sum_{j=1}^{3}\left\{\sigma_{F}\right\}_{i j, j}=-\sum_{j=1}^{3}\left(\partial p_{F} / \partial x_{j}\right) \delta_{i j}=-\partial p_{F} / \partial x_{i}$. Con- 
sequently, Eq. (9) is rewritten as

$$
\rho_{2} \frac{\partial^{2} \mathbf{u}_{2}}{\partial t^{2}}+\operatorname{grad} p_{F}=\mathbf{0} \quad, \mathbf{x} \in \Omega
$$

which is the linearized Euler equation for an acoustic fluid (see Eq. (10)). As a consequence, Eqs. (12) to (14) (for an acoustic fluid) are equivalent to the following equations (for an elastic solid)

$$
\begin{gathered}
\rho_{2} \frac{\partial^{2} \mathbf{u}_{2}}{\partial t^{2}}-\operatorname{div} \sigma_{F}=\mathbf{0} \quad, \mathbf{x} \in \Omega_{2} \\
\sigma_{F} \cdot \mathbf{n}=\mathbf{0} \quad, \mathbf{x} \in \boldsymbol{\Gamma}_{\mathbf{2}} \\
\mathbf{u}_{2} \cdot \mathbf{n}=\mathbf{u} \cdot \mathbf{n} \quad, \mathbf{x} \in \Gamma
\end{gathered}
$$

with $\left\{\sigma_{F}(\mathbf{x}, t)\right\}_{i j}=\sum_{k, h=1}^{3} c_{F, i j k h} \varepsilon_{F, k h}(\mathbf{x}, t)$ and $c_{F, i j k h}=c_{2,0}^{2} \rho_{0,2} \delta_{i j} \delta_{k h}$. As for the elastic solid layer, we can introduce an elasticity matrix $\left[C^{F}\right]$ whose entries are the components $c_{F, i j k h}$ of the elasticity tensor of the fluid medium. All entries of $\left[C^{F}\right]$ are zero except $\left[C^{F}\right]_{11},\left[C^{F}\right]_{12},\left[C^{F}\right]_{13},\left[C^{F}\right]_{21},\left[C^{F}\right]_{22}$, $\left[C^{F}\right]_{23},\left[C^{F}\right]_{31},\left[C^{F}\right]_{32}$ and $\left[C^{F}\right]_{33}$ that are all equal to $\rho_{0,2} c_{2}^{2}$.

\section{Simplified mean model for the elastic solid layer with a gradient model}

A healthy cortical bone can be viewed as a porous material. In this case, it has reported that Haversian/Volkmann's canals are the major portions of the total porosity with a mean diameter on an order of $50 \mu \mathrm{m}$ [15]. However, in case of bone diseases, due to a degradation of the cortical bone in the neighborhood of the marrow, in this region of the bone, the pore sizes are enough large and are not small with respect to the thickness of the cortical layer. In addition, the number of pores in this region is too small. Consequently, the usual porous medium theory $[1,2,3]$ cannot be applied. It 
should be noted that the pore sizes increase along the transverse direction $x_{3}$ and the gradient of the mean elasticity properties at mesoscale is such that, in the neighborhood of interface $\Sigma_{2}$, the cortical material is mostly made up of the same acoustic fluid than the one in domain $\Omega_{2}$. Hereafter, we then propose an approach that allows the modeling of the mean elasticity matrix, $\left[C\left(x_{3}\right)\right]$, to be still constructed. Let $a$ and $b$ such that $-h \leq b<a \leq 0$ (see Fig. 1). For all $x_{3}$ in $[a, 0]$, the mean model of material at mesoscale in the elastic solid layer is assumed to be a homogeneous transverse isotropic medium, and for all $x_{3}$ in $[-h, b]$ is assumed to be the same acoustic fluid than in the medium $\Omega_{2}$ (note that $a$ and $b$ are generally unknown and must be identified solving an inverse problem). Consequently: (i) for all $x_{3}$ in $[a, 0]$, we have $\left[C\left(x_{3}\right)\right]=\left[C^{S}\right]$ and $\rho\left(x_{3}\right)=\rho^{S}$; (ii) for all $x_{3}$ in $[-h, b]$ we have $\left[C\left(x_{3}\right)\right]=\left[C^{F}\right]$ and $\rho\left(x_{3}\right)=\rho_{0,2}$, that is to say, we have the properties of acoustic fluid layer $\Omega_{2}$. In the interval $[b, a]$, we introduce a gradient such that, for all $x_{3}$ in $[b, a]$

$$
\begin{gathered}
{\left[C\left(x_{3}\right)\right]=\left(1-f\left(x_{3}\right)\right)\left[C^{S}\right]+f\left(x_{3}\right)\left[C^{F}\right],} \\
\rho\left(x_{3}\right)=\left(1-f\left(x_{3}\right)\right) \rho^{S}+f\left(x_{3}\right) \rho_{0,2},
\end{gathered}
$$

where $f\left(x_{3}\right)=c_{0}+c_{1} x_{3}+c_{2} x_{3}^{2}+c_{3} x_{3}^{3}$ in which $c_{0}=a^{2}(a-3 b) /(a-b)^{3}$, $c_{1}=6 a b /(a-b)^{3}, c_{2}=-3(a+b) /(a-b)^{3}$ and $c_{3}=2 /(a-b)^{3}$. This model is such that $[C(a)]=\left[C^{S}\right],[C(b)]=\left[C^{F}\right]$ and for $x_{3}=a$ or $x_{3}=b$, $\partial\left[C\left(x_{3}\right)\right] / \partial x_{3}=0$ and $\partial \rho\left(x_{3}\right) / \partial x_{3}=0$. It should be note that, firstly the mean model proposed only depends on two parameters which are $a$ and $b$ and secondly, the mean thickness of the elastic solid layer at the mesoscale modeling is $|b| \leq h$. 


\section{Prior probabilistic model of the elasticity matrix at the mesoscale modeling}

At the mesoscale modeling, the cortical bone constituting the elastic solid layer is a heterogeneous anisotropic material for which the elasticity properties field is modeled by a matrix-valued random field $[\mathbf{C}]=\left\{\left[\mathbf{C}\left(x_{3}\right)\right], x_{3} \in\right.$ $[b, 0]\}$. The prior probabilistic model of $[\mathbf{C}]$ is chosen in the ensemble of tensorvalued random field adapted to elliptic operator, defined in Soize [22, 23]. This probability model of the uncertain parameters are constructed by using the maximum entropy principle $[21,11,12]$. For all $b \leq x_{3} \leq 0,\left[\mathbf{C}\left(x_{3}\right)\right]$ is a positive-definite random matrix which is written as

$$
\left[\mathbf{C}\left(x_{3}\right)\right]=\left[L\left(x_{3}\right)\right]^{T}\left[\mathbf{G}\left(x_{3}\right)\right]\left[L\left(x_{3}\right)\right]+\left[C_{0}\left(x_{3}\right)\right],
$$

in which the deterministic matrix $\left[C_{0}\left(x_{3}\right)\right]$ is positive-definite and the matrix $\left[\mathbf{G}\left(x_{3}\right)\right]$ is a positive-definite random matrix; these two matrices are defined below. In the definition of $\left[\mathbf{C}\left(x_{3}\right)\right]$, an upperscript $T$ designates the transpose operator. By construction, we have

$$
E\left\{\left[\mathbf{C}\left(x_{3}\right)\right]\right\}=\left[C\left(x_{3}\right)\right] \quad, \quad \forall x_{3} \in[b, 0],
$$

in which $[C]=\left\{\left[C\left(x_{3}\right)\right], x_{3} \in[b, 0]\right\}$ is the mean value field defined in the previous section and the operator $E\{\cdot\}$ denotes the mathematical expectation. Positive-definite matrix $\left[C_{0}\left(x_{3}\right)\right]$ must be such that, for all $x_{3}$ in $[b, 0]$, $\left[C\left(x_{3}\right)\right]-\left[C_{0}\left(x_{3}\right)\right]$ is positive-definite. The $6 \times 6$ upper triangular matrix $\left[L\left(x_{3}\right)\right]$ corresponds to the Cholesky decomposition of the positive-definite matrix $\left[C\left(x_{3}\right)\right]-\left[C_{0}\left(x_{3}\right)\right]$, that is to say $\left[C\left(x_{3}\right)\right]-\left[C_{0}\left(x_{3}\right)\right]=\left[L\left(x_{3}\right)\right]^{T}\left[L\left(x_{3}\right)\right]$. The matrix-valued random field $[\mathbf{G}]=\left\{\left[\mathbf{G}\left(x_{3}\right)\right], x_{3} \in \mathbb{R}\right\}$ is defined as a 
non-linear mapping of 21 independent second-order centered homogeneous Gaussian random fields $U_{j j^{\prime}}=\left\{U_{j j^{\prime}}\left(x_{3}\right), x_{3} \in \mathbb{R}\right\}$ with $1 \leq j \leq j^{\prime} \leq 6$. The explicit expression of this non-linear mapping can be found in Soize [22, 23] and are written as

$$
\begin{gathered}
{\left[\mathbf{L}_{\mathbf{G}}\left(x_{3}\right)\right]_{j j^{\prime}}=\sigma_{n} U_{j j^{\prime}}\left(x_{3}\right)} \\
{\left[\mathbf{L}_{\mathbf{G}}\left(x_{3}\right)\right]_{j j}=\sigma_{n} \sqrt{2 h\left(\alpha_{j}, U_{j j}\left(x_{3}\right)\right)}}
\end{gathered}
$$

for all $1 \leq j<j^{\prime} \leq n$, where the random upper triangular matrix $\left[\mathbf{L}_{\mathbf{G}}\left(x_{3}\right)\right]$ corresponds to the Cholesky decomposition of the random positive-definite matrix $\left[\mathbf{G}\left(x_{3}\right)\right]=\left[\mathbf{L}_{\mathbf{G}}\left(x_{3}\right)\right]^{T}\left[\mathbf{L}_{\mathbf{G}}\left(x_{3}\right)\right]$ and in which $\sigma_{n}=\delta(n+1)^{-1 / 2}, \alpha_{j}=$ $(n+1) /\left(2 \delta^{2}\right)+(1-j) / 2$ and $h(\alpha, U)$ is a Gamma random variable with parameter $\alpha$ written as $h(\alpha, u)=F_{\Gamma_{\alpha}}^{-1}\left(F_{U}(u)\right)$ where $F_{U}$ and $F_{\Gamma_{\alpha}}$ are the cumulative distribution functions of a normalized Gaussian random variable $U$ and a Gamma random variable $\Gamma_{\alpha}$ with parameter $\alpha$. The 21 independent stochastic germs $U_{j j^{\prime}}$ are then defined by 21 autocorrelation functions $R_{U_{j j^{\prime}}}(\xi)=E\left\{U_{j j^{\prime}}\left(x_{3}+\xi\right) U_{j j^{\prime}}\left(x_{3}\right)\right\}$ which are all chosen equal to a same unique function $(2 \ell / \pi \xi)^{2} \sin ^{2}(\pi \xi / 2 \ell)$ depending only on a spatial correlation length denoted by $\ell$. The random field $[\mathbf{G}]$ also depends on an additional parameter $\delta$ independent of $x_{3}$ such that $E\left\{\left\|\left[\mathbf{G}\left(x_{3}\right)\right]\right\|_{F}^{2}\right\}=6\left(\delta^{2}+1\right)$. It is shown that the statistical fluctuations of random matrix $\left[\mathbf{C}\left(x_{3}\right)\right]$ are controlled by the dispersion parameter $\delta_{C}\left(x_{3}\right)$ defined by $\delta_{C}\left(x_{3}\right)^{2}=E\left\{\|\left[\mathbf{C}\left(x_{3}\right)\right]-\right.$ $\left.\left[C\left(x_{3}\right)\right] \|_{F}^{2}\right\} /\left\|\left[C\left(x_{3}\right)\right]-\left[C_{0}\left(x_{3}\right)\right]\right\|_{F}^{2}$ (in which $\|\cdot\|_{F}$ is the Frobenius norm), and which is related parameter $\delta$ by the following equation

$$
\delta_{C}\left(x_{3}\right)=\frac{\delta}{\sqrt{7}}\left(1+\frac{\left(\operatorname{tr}\left[C\left(x_{3}\right)\right]-\operatorname{tr}\left[C_{0}\left(x_{3}\right)\right]\right)^{2}}{\left(\operatorname{tr}\left[C\left(x_{3}\right]-\left[C_{0}\left(x_{3}\right)\right]\right)^{2}\right.}\right)^{1 / 2},
$$

where tr designates the trace operator. 
Finally, the spatial correlation length $\ell_{C}$ of random field $[\mathbf{C}]$ is defined by

$$
\ell_{C}=\int_{0}^{+\infty}\left|r_{c}(\xi)\right| d \xi
$$

in which

$$
r_{c}(\xi)=\frac{\operatorname{tr} E\left\{\left(\left[\mathbf{C}\left(x_{3}+\xi\right)\right]-\left[C\left(x_{3}\right]\right)\left(\left[\mathbf{C}\left(x_{3}\right)\right]-\left[C\left(x_{3}\right)\right]\right]\right)\right\}}{E\left\{\left\|\left[\mathbf{C}\left(x_{3}\right)\right]-\left[C\left(x_{3}\right)\right]\right\|_{F}^{2}\right\}} .
$$

It is deduced from the previously quoted papers that the probability density functions $p_{[\mathbf{C}]}$ of random matrix $\left(\left[\mathbf{C}\left(x_{3}\right)\right]\right.$ with respect to the measure $d \tilde{A}=$ $2^{n(n-1) / 4} \prod_{1 \leq i \leq j \leq n}[A]_{i j}$ (with $n=6$ ) on the set $\mathbb{M}^{+}$of the symmetric positive $n \times n$ real matrices is then written as

$$
\begin{aligned}
p_{\left[\mathbf{C}\left(x_{3}\right)\right]}\left([A] ; x_{3}\right) & =\mathbb{I}_{\mathbb{M}^{+}}\left([A]-\left[C_{0}\left(x_{3}\right)\right]\right) C \operatorname{det}\left([A]-\left[C_{0}\left(x_{3}\right)\right]\right)^{\lambda-1} \\
& \times \exp \left\{-\frac{n-1+2 \lambda}{2} \operatorname{tr}\left(\left(\left[C\left(x_{3}\right)\right]-\left[C_{0}\left(x_{3}\right)\right]\right)^{-1}\left([A]-\left[C_{0}\left(x_{3}\right)\right]\right)\right\},\right.
\end{aligned}
$$

where $\mathbb{I}_{\mathbb{M}^{+}}([A])$ is equal to 1 if $[A]$ belongs to $\mathbb{M}^{+}$and is equal to zero if $[A]$ does not belong to $\mathbb{M}^{+} ; \operatorname{tr}(\cdot)$ is the trace operator; $C$ is a normalization constant; $\lambda$ is a positive real parameters that depends on the statistical fluctuation of random matrices $\left[\mathbf{C}\left(x_{3}\right)\right]$. It should be noticed that random field $[\mathbf{C}]$ is non Gaussian.

For the application to the cortical bone, we do not have any information concerning matrix $\left[C_{0}\left(x_{3}\right)\right]$ which is only introduced to preserve the ellipticity property of the stiffness operator. This matrix can be chosen, for $x_{3}$ in $[b, 0]$, as $\left[C_{0}\left(x_{3}\right)\right]=\eta_{0}\left[C\left(x_{3}\right)\right]$ in which $0<\eta_{0}<1$. In this case, $\eta_{0}$ can be chosen very small if no information concerning $\left[C_{0}\left(x_{3}\right)\right]$ is available.

With such a stochastic modeling, the displacement field of the elastic solid layer and the two acoustic pressure fields of the acoustic fluid layers are random fields denoted by $\mathbf{U}, P_{1}$ and $P_{2}$. 


\section{Application}

In a previous work [9], the components of matrix $\left[C^{S}\right]$ has been identified with an experimental database using measurements of the first arriving signal with the axial transmission technique whose experimental configuration is described by Fig. 2 (see $[5,6,8,10,17])$. A coupling gel is applied at the interface between the device and the skin of the patient. Each transmitter generates an acoustical impulse in the ultrasonic range that propagates in the coupling gel, the skin, the muscle, the cortical bone and the marrow. The

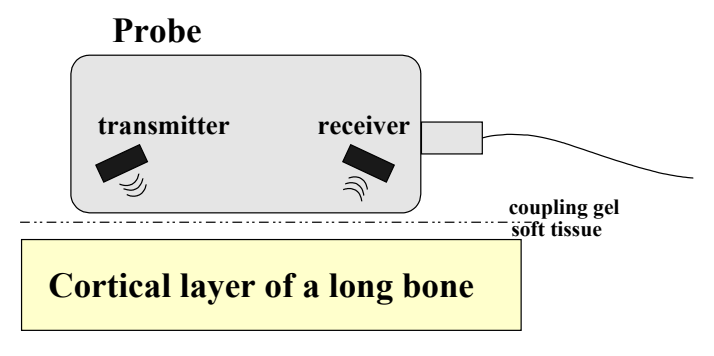

Figure 2: Experimental configuration. Modifier le second schéma

axial transmission technique consists in recording these signals at the $n_{R}=11$ receivers located in the device. The first arriving signal (FAS) is considered. Following the signal processing method used with the experimental device (see $[5,6,8,10,17])$, the velocity of FAS is determined from the time of flight of the first extremum of the contribution. This experimental database allows the components of matrix $\left[C^{S}\right]$ to be identified (see [9]) and we obtained $\rho^{S}=1598.8 \mathrm{~kg} . \mathrm{m}^{-3}, e_{L}=17.717 \mathrm{GPa}, \nu_{L}=0.3816, g_{L}=4.7950 \mathrm{GPa}, e_{T}=$ $9.8254 \mathrm{GPa}, \nu_{T}=0.4495$ and $\delta_{C}(0)=0.1029$ with $h=4 \mathrm{~mm}$. The material properties of both fluids are the ones defined in Desceliers et al. [9] which are recalled here $\rho_{0,1}=\rho_{0,2}=1000 \mathrm{~kg} \cdot \mathrm{m}^{-3}$ and $c_{0,1}=c_{0,2}=1500 \mathrm{~m} \cdot \mathrm{s}^{-1}$. The 
acoustic line source is located at $x_{1}^{S}=0$ and $x_{3}^{S}=2 \times 10^{-3} \mathrm{~m}$.

Using Eq. (21) yields $\delta=0.0575$. We are interested by the propagation of the uncertainties in the first fluid layer $\Omega_{1}$ for the cortical bone system in the context of the axial transmission technique. We then introduce the random variable $Q$ defined by

$$
Q=\int_{0}^{T} \sum_{k=1}^{n_{R}}\left|P_{2}\left(t, x_{1}^{k}\right)\right|^{2} d t
$$

where $T$ is the duration of an experimental signal and $x_{1}^{k}$, with $k=1, \ldots, n_{R}$ are the positions of the receivers along direction $\mathbf{e}_{1}$. Let $q \mapsto p_{Q}(q ; a, b, \ell)$ be the probability density function of random variable $Q$. In Fig. 3, the graph of $x_{3} \mapsto \delta_{C}\left(x_{3}\right)$ is shown with $a=0, b=-h$ (thin solid line) and $a=-h / 2, b=-h$ (thick solid line) and $a=0, b=-h / 2$ (dashed thin line). It can been seen that the value of the dispersion coefficient $\delta_{C}\left(x_{3}\right)$ of the random matrix $\left[\mathbf{C}\left(x_{3}\right)\right]$ decreases when one moves to the neighborhood of the interface between the elastic solid layer and the acoustic fluid layer. The constitutive equations of the material go to the constitutive equations of the fluid in $\Omega_{2}$ that is not uncertain. In Fig. 4, the graph of $q \mapsto p_{Q}(q ; a, b, \ell)$ is shown in logscale with $a=0, b=-h, \ell=h / 10$ (thick solid line), with $a=-h / 2, b=-h, \ell=h / 10$ (thin solid line), with $a=0, b=-h, \ell=h / 20$ (thick dashed line), with $a=-h / 2, b=-h, \ell=h / 20$ (thin dashed line). It can be seen that the probability density function is sensitive with respect to the $a, b$ and the spatial correlation length $\ell$. 


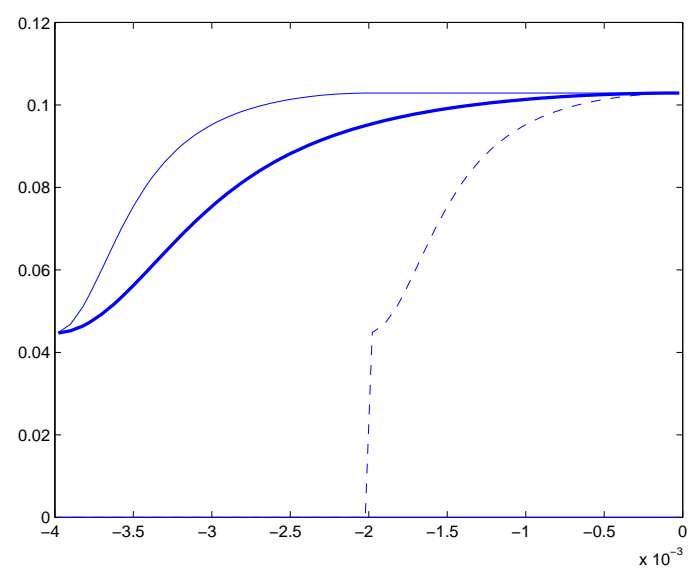

Figure 3: Graph of $x_{3} \mapsto \delta_{C}\left(x_{3}\right)$ with $a=0, b=-h$ (thin solid line) and $a=-h / 2, b=-h$ (thick solid line) and $a=0, b=-h / 2$ (dashed thin line)

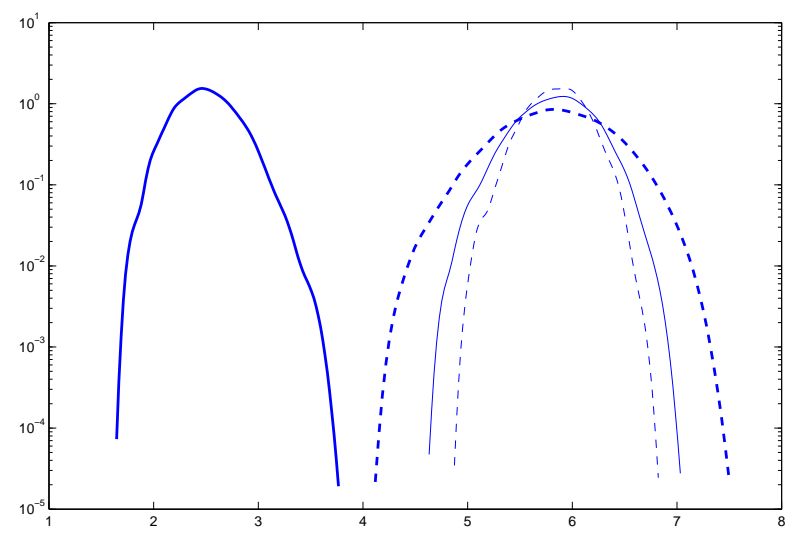

Figure 4: Graph of $q \mapsto p_{Q}(q ; a, b, \ell)$ in logscale with $a=0, b=-h, \ell=h / 10$ (thick solid line), with $a=-h / 2, b=-h, \ell=h / 10$ (thin solid line), with $a=-h / 2, b=-h$, $\ell=h / 20$ (thin dashed line), with $a=-h / 2, b=-h, \ell=h$ (thick dashed line). 


\section{Conclusion}

In this paper we have considered the transient dynamical response of a multilayer system submitted to an impulse in the ultrasonic range. The application concerns a biomechanical system: the human cortical bone. This system is really tricky to be modelled due to the lack of knowledge on its micro-structure. For such a system, the micro-structure can be altered near its interface with the marrow due to bone diseases. A gradient of porosity is then observed in the thickness direction but, in this case, none of the usual theories of porous materials can be applied. This is the reason why we have proposed a simple model of the elasticity tensor for media with a gradient of the porosity in order to take into account the alterations of the cortical bone micro-structure. Thus, inside the elastic solid layer, the constitutive equation of the elastic solid goes to the constitutive equation of the fluid (the marrow). Then, in order to improve the predictability of this simplified model, we have taken into account the uncertainties by substituting the elasticity tensor with a random field for which the probabilistic model has been constructed using the maximum entropy principle. An application has been proposed to study the propagation of these uncertainties on the acoustic pressure field inside the first fluid domain (the skin). Results show that the total energy of the random pressure pressure field is very sensitive to the gradient and the spatial correlation length of the random elasticity tensor in the cortical layer. Consequently, experimental measurements in the context of the axial transmission technique can be used in order to identify the parameters of this probabilistic model. 


\section{Acknowledgments}

This research was supported by the "Agence Nationale de la Recherche", Contract TYCHE, ANR-2010-BLAN-0904.

\section{References}

[1] M. A. Biot. Theory of propagation of elastic waves in a fluid-saturated porous solid. I. Low-frequency range. Journal of the Acoustical Society of America, 28(2):168-178, 1956.

[2] M. A. Biot. Theory of propagation of elastic waves in a fluid-saturated porous solid. II. Higher frequency range. Journal of the Acoustical Society of America, 28(2):179-191, 1956.

[3] M. A. Biot. Mechanics of deformation and acoustic propagation in porous media. Journal of Applied Physics, 33(4):1482-1498, 1962.

[4] E. Bossy, M. Talmant, and P. Laugier. Bi-directional axial transmission can improve accuracy and precision of ultrasonic velocity measurement in cortical bone: a validation on test material. IEEE Trans. Ultrason. Ferroelectr. Freq. Control., 51(1):71-79, 2004.

[5] E. Bossy, M. Talmant, and P. Laugier. Three-dimensional simulations of ultrasonic axial transmission velocity measurement on cortical bone models. Journal of the Acoustical Society of America, 115(5):2314-2324, 2004 .

[6] E. Bossy, M. Talmant, F. Peyrin, L. Akrout, P. Cloetens, and P. Laugier. An in vitro study of the ultrasonic axial transmission technique at the 
radius: 1-mhz velocity measurements are sensitive to both mineralization and intracortical porosity. Journal of Bone and Mineral Research, 19(9):1548-1556, 2004.

[7] V. Bousson, A. Meunier, C. Bergot, E. Vicaut, M.A. Rocha, M.H. Morais, A.M. Laval-Jeantet, and J.D. Laredo. Distribution of intracortical porosity in human midfemoral cortex by age and gender. $J$. Bone Mineral Res., 16(17):1308-1317, 2001.

[8] E. Camus, M. Talmant, G. Berger, and P. Laugier. Analysis of the axial transmission technique for the assessment of skeletal status. Journal of the Acoustical Society of America, 108(96):3058-3065, 2000.

[9] C. Desceliers, C. Soize, M. Grimal, Q.and Talmant, and S. Naili. Determination of the random anisotropic elasticity layer using transient wave propagation in a fluid-solid multilayer: Model and experiments. Journal of the Acoustical Society of America, 125(4, Part 1):2027-2034, 2008.

[10] Q. Grimal, K. Raum, C. Desceliers, C. Soize, G. Haiat, S. Naili, M. Talmant, and P. Laugier. Stochastic simulation of the axial transmission technique based on a multi-scale model of bone material properties. Halle, Allemagne, 2007. 2nd European Symposium on ultrasonic characterization of bone. July 19-20.

[11] E.T. Jaynes. Information theory and statistical mechanics. The Physical Review, 106(4):620-630, 1957.

[12] E.T. Jaynes. Information theory and statistical mechanics. II. The Physical Review, 108(2):171-190, 1957. 
[13] G. Lowet and G. Van der Perre. Ultrasound velocity measurements in long bones: measurement method and simulation of ultrasound wave propagation. Journal of Biomechanics, 29:1255-1262, 1996.

[14] K. Macocco, Q. Grimal, S. Naili, and C. Soize. Elastoacoustic model with uncertain mechanical properties for ultrasonic wave velocity prediction; application to cortical bone evaluation. Journal of the Acoustical Society of America, 119(2):729-740, 2006.

[15] R.B. Martin, D.B. Burr, and N.A. Sharkey. Skeletal Tissue Mechanics. Springer-Verlag, 1998.

[16] S Naili, M.B. Vu, Q. Grimal, M. Talmant, C. Desceliers, C. Soize, and G. Haiat. Influence of viscoelastic and viscous absorption on ultrasonic wave propagation in cortical bone: Application to axial transmission. Journal of the Acoustical Society of America, 127(4):2622-2634, 2010.

[17] K. Raum, I. Leguerney, F. Chandelier, E. Bossy, M. Talmant, A. Saied, F. Peyrin, and P. Laugier. Bone microstructure and elastic tissue properties are reflected in QUS axial transmission measurements. Ultrasound in Medicine and Biology, 31(9):1225-1235, 2005.

[18] V. Sansalone, S. Naili, V. Bousson, C. Bergot, F. Peyrin, J. Zarka, J. D. Laredo, and G. Haiat. Determination of the heterogeneous anisotropic elastic properties of human femoral bone: from nanoscopic to organ scale. J. Biomech., 43:1857-1863, 2010.

[19] M. Sasso, G. Haiat, Y. Yamato, S. Naili, and M. Matsukawa. Frequency 
dependence of ultrasonic attenuation in bovine cortical bone: an in vitro study. Ultrasound Med. Biol., 33(12):1933-1942, 2007.

[20] M. Sasso, G. Haiat, Y. Yamato, S. Naili, and M. Matsukawa. Dependence of ultrasonic attenuation on bone mass and microstructure in bovine cortical bone. J. Biomech., 41(2):347-355, 2008.

[21] C.E. Shannon. A mathematical theory of communication. Bell System Technology Journal, 27:379-423, 1948.

[22] C. Soize. Non-Gaussian positive-definite matrix-valued random fields for elliptic stochastic partial differential operators. Computer Methods in Applied Mechanics and Engineering, 195:26-64, 2006.

[23] C. Soize. Tensor-valued random fields for meso-scale stochastic model of anisotropic elastic microstructure and probabilistic analysis of representative volume element size. Probabilistic Engineering Mechanics, 23: 307-323, 2008. 